\title{
Control of Vortex Shedding behind a Circular Cylinder in an Electrically Low-Conducting Fluid-Revised
}

\author{
Ying $\mathrm{He}^{\mathrm{a}}$, Zhihua Chen ${ }^{\mathrm{b}}$, Xinmin Yang ${ }^{\mathrm{c}}$, Wenjun $\mathrm{Yi}^{\mathrm{d}}$ \\ School of Nanjing University of Science and Technology, Nanjing 210094, China \\ aheying2172@foxmail.com, b916047597@qq.com, cyxm@mail.njust.edu.cn, dyiwenjun0@163.com
}

Keywords: Vortex Shedding, Flow Control, Flow Separation, Lorentz Force.

\begin{abstract}
A closed-loop control algorithm has been developed for suppressing the vortex shedding of flow past a circular cylinder in an electrically low-conducting fluid (e.g. seawater). The intent is to avoid both vortex shedding and flow separation from the body, which is achieved through the introduction of localized electromagnetic forces (Lorentz forces) generated by an array of permanent magnets and electrodes on the surface of the circular cylinder. More specifically, the closed-loop control has been derived from the equations capable of determining at all times the intensity of the Lorentz force in order to control the flow. This is accomplished first, independently of the flow (open loop algorithm) and second, based on some partial flow information measured on the surface of the solid body.
\end{abstract}

\section{Introduction}

Vortex shedding of an isolated circular cylinder is an important and very well investigated benchmark problem in fluid mechanics ${ }^{[1]}$. Due to its academic interest and practical importance, in which the mechanism involved is essential to the flow-induced vibrations, fluid/structure interaction phenomena and acoustic noise in many situations, it also remains one of the most investigated and best understood bluff body wake problems. Many investigations have so far been concentrated on suppressing vortex shedding from bluff bodies ${ }^{[2-5]}$, with the using of splitter plate, rotary oscillation of cylinder, insertion of additional vortices etc., in order to achieve turbulent boundary layer control, transition delay and drag reduction.

In an electrically conducting fluid (such as sea water), it is possible, through the motion of a conducting material in a magnetic field, to generate an electromotive force and cause an electric current of density to flow. The current induces its own magnetic field and organizes a volume force distribution inside the fluid. The induced currents, together with the external magnetic field, generate a Lorentz force, which, in turn, influences the fluid flow. The main advantage of the control strategy for electrically conducting fluids is that the Lorentz force acts on a volume of the flow, and it may be possible to tune the force to act only in specific regions of the flow. Many techniques using electric and magnetic fields have been proposed in weakly conducting fluids ${ }^{[6-9]}$. Although many experiments and numerical analyses have been carried out for such MHD and EMHD flows, little is known about the instability and transition scenarios except for the general belief that magnetic fields have a damping influence. Furthermore, there has not been any investigation on the design of flow control based on some instantaneous flow information for these flows. The purpose of this research is to develop a closed-loop control algorithm for manipulating wake flows past a fixed cylinder at $\mathrm{Re}=100$ in an electrically low-conducting fluid. Our goal is to avoid flow separation from the surface of the body and fully suppress the vortex shedding behind the circular cylinder.

\section{Governing Equations and Numerical Method}

The Lorentz force in an electrically conducting medium of conductivity $\sigma$ can be written by the vector product of the electric current $j$ with the magetic field $B, F=j \times B$. The current density $j$ is given by Ohm's law $\mathrm{j}=\sigma(\mathrm{E}+\mathrm{u} \times \mathrm{B})$. In low-conducting fluids like seawater, the induced electrical current $\mathrm{j}=\sigma \mathrm{U} \times \mathrm{B}$, are generally too small to produce any noticeable effect compared to the current associated 
with the electric field, and is thus neglected. The Lorentz force then reduces to $\mathrm{F}=\sigma \mathrm{E} \times \mathrm{B}$, which has a streamwise component only. Consider an incompressible electrically conducting viscous flow governed by the time-dependent N-S equations and the continuity equation:

$$
\frac{\partial \mathbf{V}}{\partial t}+(\mathbf{V} \cdot \nabla) \mathbf{V}=-\nabla p+\frac{2}{\operatorname{Re}} \nabla^{2} \mathbf{V}+N \mathbf{F}
$$

$\nabla \cdot \mathbf{V}=0$

Where $\operatorname{Re}=\frac{2 u_{\infty} a}{v}, N=\frac{j_{0} B_{0} a}{\rho u_{\infty}^{2}} . \mathrm{N}$ denotes the interaction parameter; $\mathrm{F}$ refers to the dimensionless Lorentz force. The applied electrical current density $j_{0}=\sigma E_{0}$, E0 denotes the electric field and B0 is the magnetic field.

Introducing the exponential-polar coordinates system $(\xi, \eta), r=e^{2 \pi \xi}, \theta=2 \pi \eta$, and defining the stream function $\psi$ and vorticity $\Omega$ as $\frac{\partial \psi}{\partial \eta}=U_{r}=H^{\frac{1}{2}} u_{r},-\frac{\partial \psi}{\partial \xi}=U_{\theta}=H^{\frac{1}{2}} u_{\theta}, \Omega=\frac{1}{r} \frac{\partial\left(r u_{\theta}\right)}{\partial r}-\frac{1}{r} \frac{\partial u_{r}}{\partial \theta}$, where $u_{r}$ and $u_{\vartheta}$ and $u_{\vartheta}$ is the projection of the velocity $\mathbf{V}$ in the direction of $r, \theta$ respectively, and $H=4 \pi^{2} e^{4 \pi \xi}$. Then the conservative equations can be written as

$$
\begin{aligned}
& H \frac{\partial \Omega}{\partial t}+\frac{\partial\left(U_{r} \Omega\right)}{\partial \xi}+\frac{\partial\left(U_{\theta} \Omega\right)}{\partial \eta}=\frac{2}{R_{e}}\left(\frac{\partial^{2} \Omega}{\partial \xi^{2}}+\frac{\partial^{2} \Omega}{\partial \eta^{2}}\right)+N H^{\frac{1}{2}}\left(\frac{\partial F_{\theta}}{\partial \xi}+2 \pi F_{\theta}\right) \\
& \frac{\partial^{2} \psi}{\partial \xi^{2}}+\frac{\partial^{2} \psi}{\partial \eta^{2}}=-H \Omega
\end{aligned}
$$

Except on the surface of the cylinder where the absence of slip is imposed, the flow is considered potential initially. For initial condition, $\mathrm{t}=0$, on the cylinder surface $\xi=0, \psi=0, \Omega=-\frac{1}{H} \frac{\partial^{2} \psi}{\partial \xi^{2}}$; away from the cylinder surface, $\xi>0, \psi=-2 \operatorname{sh}(2 \pi \xi) \sin (2 \pi \eta), \Omega=0$. The boundary condition consists of the no-slip boundary condition on the surface, and the potential flow at the outer boundary of the domain. So, on the cylinder surface $\xi=0, \psi=0, \Omega=-\frac{1}{H} \frac{\partial^{2} \psi}{\partial \xi^{2}}$; away from the surface $\xi=\xi_{\infty}$, $\psi=-2 \operatorname{sh}(2 \pi \xi) \sin (2 \pi \eta), \Omega=0$.

The numerical method consists of an Alternative-Direction Implicit (ADI) algorithm for the vorticity transport equation (3) and Fast Fourier Transform (FFT) algorithm for the (streamfunction equation) Poisson equation (4). An adaptive scheme is also developed in order to increase the efficiency of our numerical code. This scheme consists of moving the boundary used for the vorticity transport equation further away from the body as the vorticity is transported outward.

For a unit length of cylinder, the total force acting on the body can be obtained by integrating the pressure and shear stress along the body surface, while the pressure drag can be obtained from the integration of pressure along the cylinder surface. Assuming the flow moves in $x$ direction, defining the drag, lift and pressure drag coefficients as $C_{d}=\frac{F_{t x}}{\rho u_{\infty}^{2} a}, C_{l}=\frac{F_{t y}}{\rho u_{\infty}^{2} a}, C_{p d}=\frac{F_{p x}}{\rho u_{\infty}^{2} a}$. Where $F_{t x}$ and $F_{t y}$ are the projections of the total drag in $\mathrm{x}$ and $\mathrm{y}$ direction respectively and $F_{p x}$ is the projection of pressure drag in $\mathrm{x}$ direction.

\section{Procedure}

One novel closed-loop control procedure for manipulating vortex shedding behind the circular cylinder is developed using the same actuators as Weier et al ${ }^{[9]}$. There is obviously a close relation between vortex shedding and flow separation on surface of the cylinder. When the vortex shedding takes place, the separation point moves along the surface of the cylinder. From Ref. [8, 9], we know that the suppression of flow separation can suppress vortex shedding and lead to a steady flow solution. It seems, therefore, appropriate to base our sensor on detecting flow separation. Since the flow separation point $\theta^{\prime}$ is identified as the location where the shear stress on the surface of cylinder is zero, and shear stress is proportional to the vorticity, thus, the separation point can be also 
identified as a zero-vorticity point. One effective closed-loop control algorithm for manipulating wake flows is developed here, based on the detection of the flow separation point on the surface of cylinder body. The interaction parameter $\mathrm{N}$ (actuator) has been chosen to make the total pressure drag coefficient $C_{p d}=0$. That is $N=-\frac{1}{\pi R_{e}} \frac{\int_{0}^{1} \frac{\partial \Omega}{\partial \xi} \sin (2 \pi \eta) d \eta}{\int_{0}^{1} F_{\theta} \sin (2 \pi \eta) d \eta}$. The control switch has been turned on in the following manner. When the absolute value of the vorticity at a point on the rear surface of the cylinder decreases below a certain threshold $\delta(\delta>0)$, expressed as $|\Omega| \leq \delta$, the actuator is turned on. Following are the numerical results have been described by using this technique.

The electro-magnetic forces are applied at $\mathrm{t}=500$, which means that the flow has been well developed before the control. Numerical results for control algorithm are at $\mathrm{Re}=150$ with the computational step $\Delta \xi=0.004, \eta=0.002$ and $\Delta \mathrm{t}=0.005$. Fig. 1 is the instantaneous streamlines, which shows the stabilization process of vortex shedding by our developed closed-loop control. It is clear that our control strategy has a significant effect on the flow. During the initial period, vortex shedding persists but weakens. After this initial period, vortex shedding disappears completely leading to a steady reattached flow.

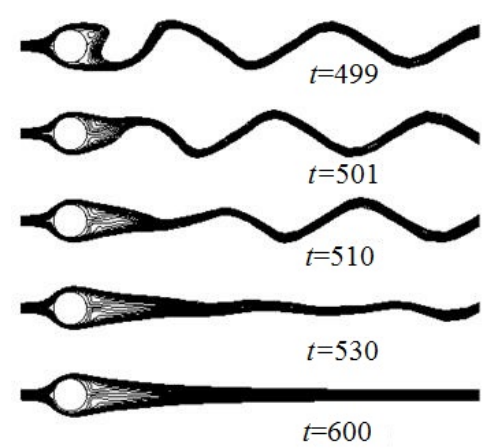

Fig.1 Flow streamlines showing the stabilization of vortex shedding by the closed-loop control. Control starts at time $\mathrm{t}=500$

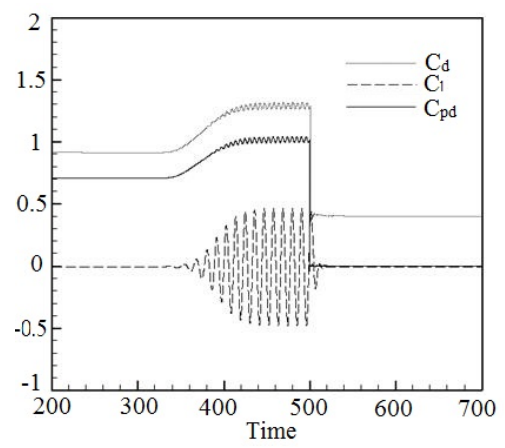

Fig.2 Time history of the drag, lift and pressure drag force coefficient with the closed-loop control technique. Control starts at $\mathrm{t}=500$.

Figure 2 displays the time history of values of the drag, lift and pressure drag force coefficients. The pressure drag and lift coefficient are successfully reduced to almost zero, while the total drag coefficient became a small constant under the action of control. Figure 3 shows the time history of the interaction parameter. After the time when the control algorithm is switched on, the interaction parameter first fluctuates and then becomes stable.

Figures 4 and 5 show the vorticity and pressure coefficient distributions along the cylinder surface. The angle $\theta$ is defined form the front to the rear stagnation point of cylinder. In particular, the vorticity distribution in Fig.4 shows that the separation is suppressed with the application of Lorentz force and that the vorticity on the surface of the cylinder is increased compared with that of the uncontrolled flow. This is due to the fact that the fluid is accelerated by the Lorentz force and that the shear stress on the surface of the cylinder is increased. Likewise, the pressure distribution decreases 
under the influence of the Lorentz force along the actuation area, while it increases at the rear stagnation point due to the reduction of the recirculation region.

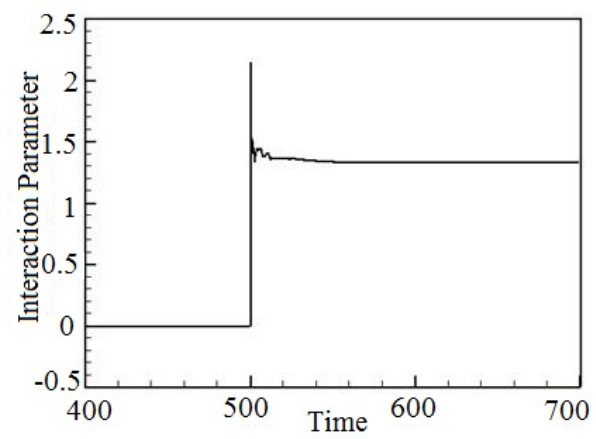

Fig.3 Time history of the interaction parameter for the flow controlled with the closed-loop control technique. Control starts at time $\mathrm{t}=500$.

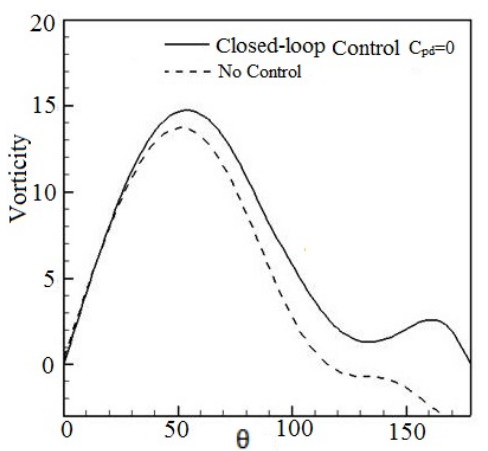

Fig.4 Vorticity distribution along the surface of the cylinder with various interaction parameters

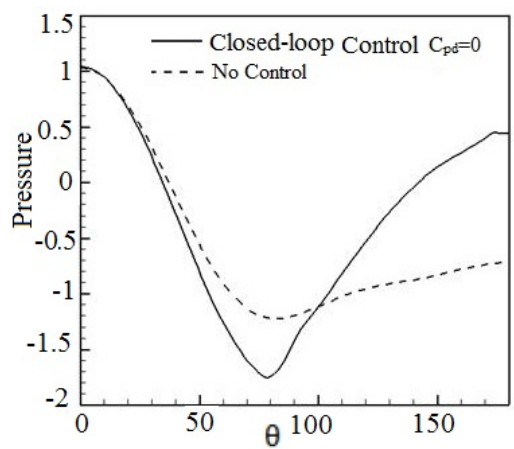

Fig.5 Pressure distribution along the surface of the cylinder with various interaction parameters

\section{Summary}

Closed-loop control procedures targeting zero pressure drag coefficient was developed and applied successfully for the suppression of vortex shedding behind a circular cylinder at $\mathrm{Re}=150$. The location of sensors was limited to the surface of the cylinder and to the measurement of the wall shear stress. The location of the actuators was determined by the information fed by the sensors, the intensity of the Lorentz force was calculated from the requirement of zero pressure drag. After a relatively short time with the control switch turned on, vortex shedding is fully suppressed and the flow becomes stable.

\section{References}

[1] Berger, E., Wille R., Periodic flow phenomena, Annual Reviews of Fluid Mechanics, 1972, 4: 313-340.

[2] Roshko, A., Perspectives on bluff body aerodynamics, J. Wind Eng. Ind. Aerod. 1993,49: 79-100. 
[3] Roussopoulos K., Monkewitz P.A., Nonlinear modelling of vortex shedding control in cylinder wakes, Physica D, 1996, 97: 264-273.

[4] Kwon, K. and Choi, H., Control of laminar vortex shedding behind a circular cylinder using splitter plates. Phys. Fluids, 1996, 8(2): 479-486.

[5] Kang, S. and Choi, H., Laminar flow past a rotating circular cylinder, Phys. Fluids, 1999, 11: 3312-3321.

[6] Ko, J.H., Dulikravich, G.S., Non-reflective boundary conditions for a consistent model of axisymmetric electro-magneto-hydrodynamic flows, Int. J. Nonl. Sci. Num. Simulation, 2000, 1(4): 247-256.

[7] Lahjomri, J., Caperan, P. and Alemany, A., The cylinder wake in a magnetic field aligned with the velocity, J. Fluid Mech., 1993, 253: 421-448.

[8] Mutschke G., Shatrov, V. and Gerbeth G., Cylinder wake control by magnetic fields in liquid metal flows. Experimental Thermal and Fluid Science, 1998, 16: 92-99.

[9] Weier T., Gerbeth G., Mutschke G., Platacis E. and Lielausis O. Experiments on cylinder wake stabilization in an electrolyte solution by means of electromagnetic forces localized on the cylinder surface. Experimental Thermal and Fluid Science, 1998, 16: 84-91. 